\title{
Application of Acoustic Directional Data for Audio Event Recognition via HMM/CRF in Perimeter Surveillance Systems
}

Syed A Yusuf ${ }^{\mathrm{a}}$, David J Brown ${ }^{\mathrm{c}}$, Alan Mackinnon ${ }^{\mathrm{a}}$

${ }^{a}$ STS Defence Ltd, Mumby Road, Gosport, PO12 1AF, United Kingdom

b Telephone: +44 (0) 2392 584222, Fax: +44 (0) 2392 529598, Email: adnan.yusuf @ sts-defence.com; adnan.yusuf@gmail.com

${ }^{c}$ Centre of Intelligent Data Solutions, 36-40 Middle Street, University of Portsmouth, Portsmouth, PO5 4BP, United Kingdom

\footnotetext{
${ }^{\text {a }}$ STS Defence Ltd, Mumby Road, Gosport, PO12 1AF, United Kingdom

b Telephone: +44 (0) 2392 584222, Fax: +44 (0) 2392 529598, Email: adnan.yusuf@sts-defence.com; adnan.yusuf@gmail.com

${ }^{\mathrm{C}}$ Centre of Intelligent Data Solutions, 36-40 Middle Street, University of Portsmouth, Portsmouth, PO5 4BP, United Kingdom
} 


\begin{abstract}
:
Audio event detection (AED) and recognition is a signal processing and analysis domain used in a wide range of applications including surveillance, home automation and behavioral assessment. The field presents numerous challenges to the current state-of-the-art due to its highly nonlinear nature. High false alarm rates (FARs) in such applications particularly limit the capabilities of vision-based perimeter monitoring systems by inducing high operator dependence. On the other hand, conventional fence-based vibration detectors and pressure-driven "taut wires" offer high sensitivity at the cost of a high FAR due to debris, animals and weather.

This work reports an audio event identification methodology implemented as a test-bed system for a surveillance application to reduce FAR, maximize blind-spot coverage and improve audio event classification accuracy. The first phase utilizes a nonlinear autoregressive classifier to locate and classify discrete audio events via an exogenous sound direction variable to improve classifier confidence. The second phase implements a time-series-based system to recognize various audio activity groups from nominal everyday sound events such as traffic and muffled speech. The discretely labeled data is thus trained with HMM and Conditional Random Field classifiers and reports a substantial improvement in classification accuracies of indoor human activities.
\end{abstract}

Keywords: Robotics, Automation, Hidden Markov Models, Conditional Random Fields, Audio Event Detection, Machine Learning, Artificial Intelligence

\title{
1 Introduction
}

The role of audio/video analytics and multi-sensor biometric systems is gaining extreme importance in the recent automation advances in the security and surveillance applications. An increasing number of perimeter security and surveillance firms are now offering multiple-sensor threat identification and classification solutions to detect unwarranted and illegal activity in fenced areas [1]. Multi-sensor surveillance, particularly vision-based monitoring systems are one of the hottest areas of research at present. The existing scientific challenge is to design and develop smart, autonomous systems that are capable of detecting and tracking dynamic objects while interpreting their activities and behaviors with a minimal false alarm rate (FAR) and operator burden [2,3]. Moreover, such multi-sensor systems are also expected to cater for potential blind-spots, low bandwidth issues and power optimization requirements in order to ensure an efficient 24/7 mode of operation [4].

Conventionally available security systems does offer a wide range of wired and wireless electronic sensors that are highly capable of detecting even a minute level of disturbance, pressure change or activity in their area of interest (AOI). Such sensors generally contain underground pressure-sensitive tubes, fence-based vibration detectors, pressure-driven "taut wires", fiber optics, infrared beam dispensers and microwave barriers [5-7]. These sensors do offer a reasonable level of accuracy however this generally results in very high FARs due to high winds, severe weather conditions, foliage and animal movement. With the advent of high-speed Ethernet connectivity, internet protocol (IP) cameras are also increasingly used to complement the overall false alarm rate (FAR) from blind sensors similar to those discussed above. Despite being considered highly efficient under human operators, a large number of these networked, visual devices induce a very high burden on 
surveillance operators which also comes at the expense of high bandwidth and power requirements. During the past two decades, increased computer hardware capabilities compensated for these shortcomings by using advanced vision analytics and machine algorithms that enable automatic detection of suspicious scene activity such as object abandonment, loitering, perimeter breach, illegal parking or unsolicited vehicle behavior in traffic flows [8-10]. Yet, this domain has also faced major challenges at almost all the phases of video analysis including object identification (presence of a vehicle or person), recognition (car type, person's sex or height) and behavioral monitoring (wrong vehicular u-turn or human gestures) $[11,12]$. Moreover, conventional video device capabilities are also seriously hampered when subjected to factors such as abrupt light intensity changes, shadows/reflections, low/zero visibility conditions or full/partial occlusion [8]. Dynamic backgrounds in the form of foliage such as moving trees or water bodies such as fountains, also introduce a high FAR. Ultimately, the majority of touch-free or non-invasive identification systems such as passive infrared (IR), Light Detection and Ranging (LIDAR) or video cameras tend to suffer from blind spot vulnerabilities [13]. This vulnerability is compensated via Pan-Tilt-Zoom (PTZ) mechanisms however this requires separate models for each of the pan position and is still vulnerable to situations where a trespasser can simply monitor camera movement and exploit it to pass from a secure area without getting detected [14-16]. Moreover, cameras that particularly use object detection systems are generally trained with background models captured at fixed camera positions which mean a slight jitter in camera position generally results in a false alarm.

Based on the shortcomings of advanced vision-based and conventional multi-sensor systems, this paper proposes a methodology to develop a real-time audio-event-identification and recognition methodology for advanced, vision-based surveillance systems. Section 2 presents an overview of existing semi/fully autonomous multi-sensor security and surveillance systems currently in operation in various industrial settings. Section 3 presents an in-depth analysis of existing audio/visual event detection algorithms. Section 4 presents the proposed system architecture. Section 5 elaborates on the proposed methodology summarized earlier in this section. Section 6 presents the underlying outcomes of the two phases before concluding the paper.

\section{A Multi-Sensor Audio-Visual Analytics Framework}

Figure 1 represents the overall architecture of a multi-sensor audio-visual threat assessment system which is the ultimate aim of the implemented test-bed which will form a part of an audio-visual perimeter monitoring system. The underlying system design adopts a hierarchical sensor utilization strategy to reduce power consumption and FAR based on operational weaknesses of some sensors in an autonomous sensor module (ASM). The components of this proposed ASM architecture are shown in Figure 1 (a) which are aimed to form an industrial, rotary smart sensor unit capable of minimizing blind-spots and assisting the mounted vision systems in an improved realization of the scene.

The overall sensor output will therefore be based upon a diverse set of motion, audio and visual feedback generated as a result of various natural or human-made disturbances from the surrounding environment. The final system thus shown in Figure 1 for holistic security assessment comprises of three core modules as follows:

- An acoustic activity sensor for audio anomaly detection

- A depth-measurement sensor for poor visibility scene processing and

- A video camera for vision-based analytics

\subsection{Scope of the current research}


The scope of this research only addresses the first part (Shown as shaded blocks in Figure 3) which is the audio event detection (AED) phase of this research. The work proposes a set of pattern recognition techniques to robustly identify the whereabouts of suspicious audio events in an environment. The term suspicious within this context is defined as the acoustic signature generating from any activity within the perimeter surrounding such as audible motion, wire-cutting, drilling or digging. Such an activity must be accurately evaluated in order to minimize false alarms. The overall system architecture of this system also includes laser range-finding (LIDAR) and camera modules as shown in Figure 2 thereby presenting a broader context in which the proposed AED methodology will fit. Based on the surveillance and security requirements, the system is aimed to address and overcome the challenges of blind spot elimination and FAR minimization.

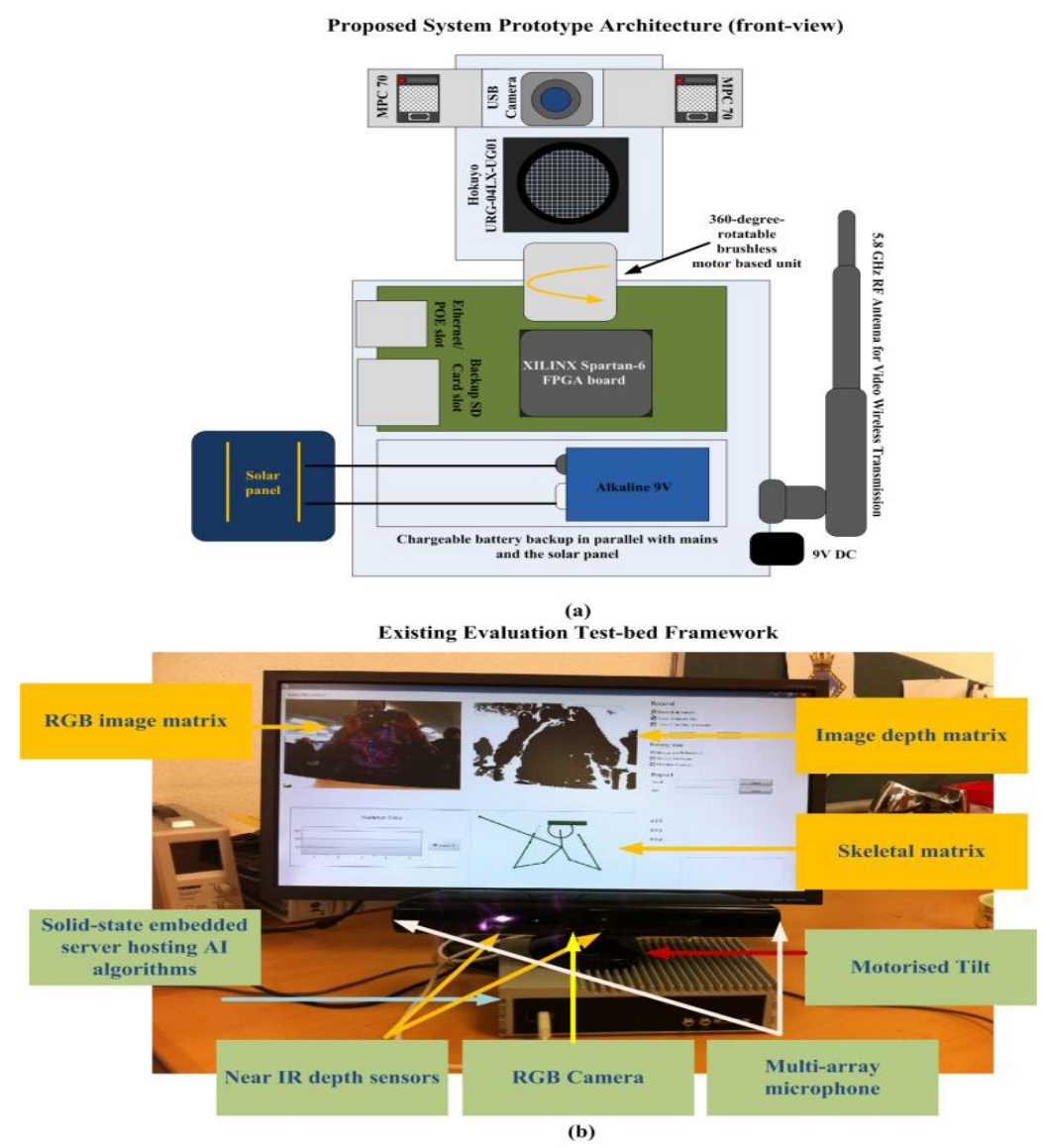

Figure 1: (a) Proposed ASM architecture with the existing test-bed system (b) to evaluate the proposed audio event identification and recognition methodology

\subsection{Proposed ASM design and the test-bed application}

A detailed theoretical overview of the proposed architecture to cover the frameworks shown in Figure 1 (a) and (b) is shown in Figure 3. The research presented in this paper is shown in the figure as shaded cells numbered 1 and 2 . The information specified via red labels in the figure demonstrates the flow-of-information between various, local decision support modules. For instance, the "sound direction and distance" information will control the panning mechanism to focus a local, low resolution, close-range RGB camera and a low-visibility-monitoring depth-sensing LIDAR sensor to detect suspicious activity via computer vision algorithms. The embedded ASM unit will physically interface with a set of low- and high-level-sensors via standard USB ports making a single 
autonomous sensor module (Figure 1 (a)). In addition to a set of similar ASMs, a single mast-based "thermal-imaging-camera (TIC)" will host a PTZ camera module to provide vision capability under zero visibility conditions. The camera directly integrates with the DSS node which collates video information from all the multi-sensor systems of individual, networked-ASMs.

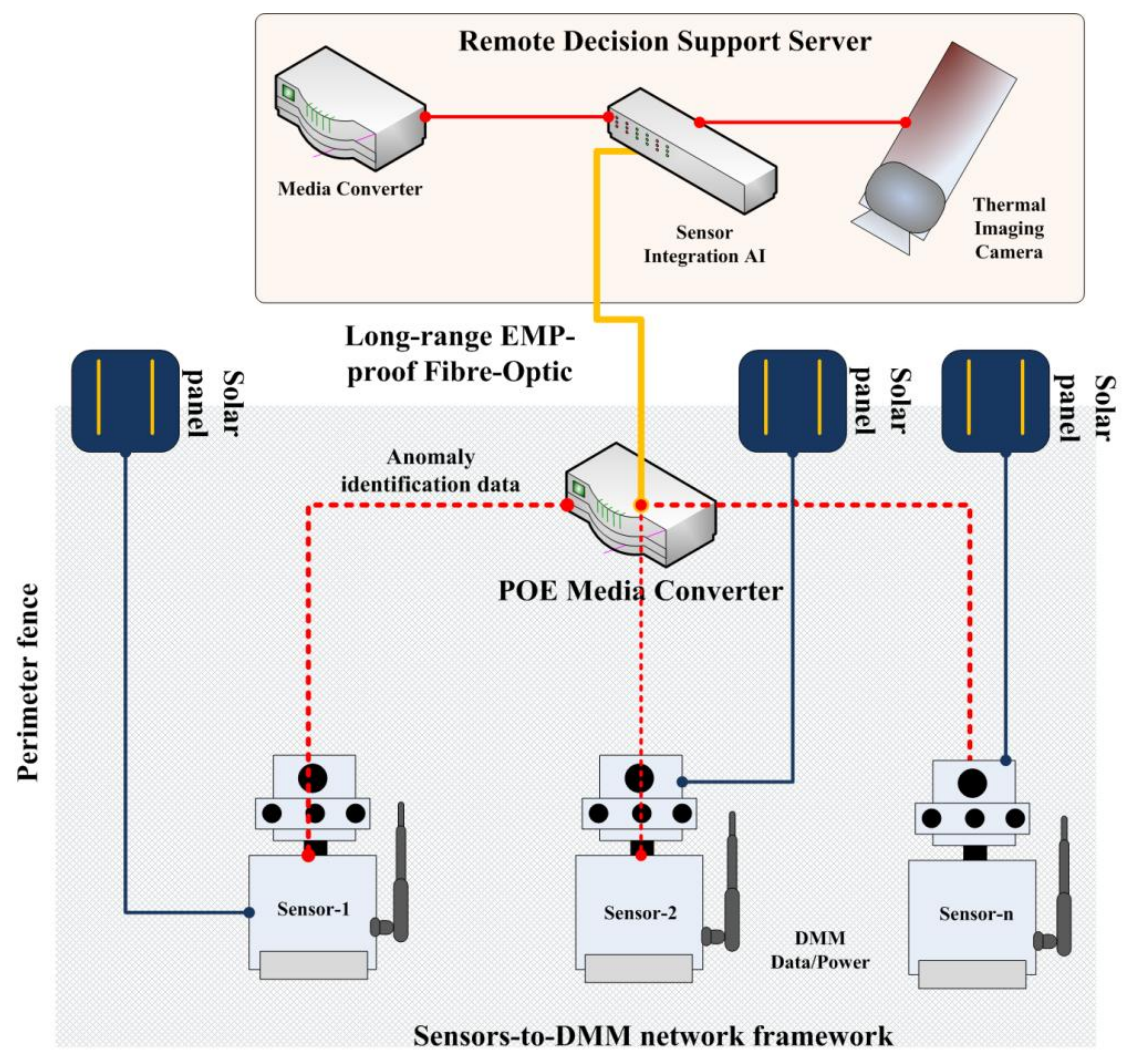

Figure 2: Overall proposed grid deployment/configuration of multiple ASMs driven via a centralized HLDSS. The Power-over-Ethernet (POE) Media Converter is proposed in this design to extend the range of IP communication

The ASMs will host a set of data processing and audio-visual pattern recognition algorithms to facilitate the required, autonomous decision making support. The decision support logic aims to utilize the strengths of each of these different sensor types in order to improve the overall detection accuracy. Moreover, this AI will also control a panning mechanism to reconfigure sensors' direction in order to reduce the FAR by eliminating surveillance blind spots. In order to achieve high performance in detection, the system aims to adopt a bottom-up approach to sensing. The most basic sensors, that include microwave motion, acoustic (sound) signature and ultrasonic range sensor, are integrated as a single module. The module acts as an early warning system (EWS) to improve the overall detection accuracy of the local video camera module. In conventional systems, it is the individual operation of these sensors that result in a high FAR and increased operator burden. A classification methodology based on the integration of these sensors is expected to improve the overall accuracy of the system. For instance, due to wire-cutting, drilling, digging or climbing, the system activates a sensor-panning mechanism to visually confirm the activity. This is done by an additional set of ASM-based video/ultrasonic sensors and an onboard computer vision object detection algorithm (Figure 3). It must be noted that the "activity recognition algorithms" is hosted over the core decision making node present on the "mast-based ASM" along with the PTZ thermal camera (Shown with gray-shaded background in Figure 3). 


\section{Theoretical Framework and System Development Methodology}

Hidden Markov Models (HMM) represent a linear sequence of time-series-driven decisions. In practice, Bayesian classifiers are useful in categorizing discrete signal events like identifying a sound type to a certain group, for instance, breaking glass or door knocks based upon the latent sound energy or other parametric variations in the audio signature of the event. However, due to their inherently discrete nature, Bayes systems cannot be used to identify collection of time-separated events; for instance, a walking-steps-sound followed by a door-opening activity or may be. Accordingly, Conditional Random Fields (CRF) can be understood as the sequenced extension of Maximum Entropy Models, indicating their discriminative modeling nature as shown in Figure 4. However, contrary to HMM, CRFs are not linearly-tied and can be structured arbitrarily. In an audio event detection and identification scope, such as that addressed in this work, audio activity is described in two different perspectives. Any typical, real-world audio environment is composed of two sets of events with one being the individual sounds such as talking, typing, etc. The second perspective relates to the temporal nature of these discrete events when joined together and considered in a holistic manner.

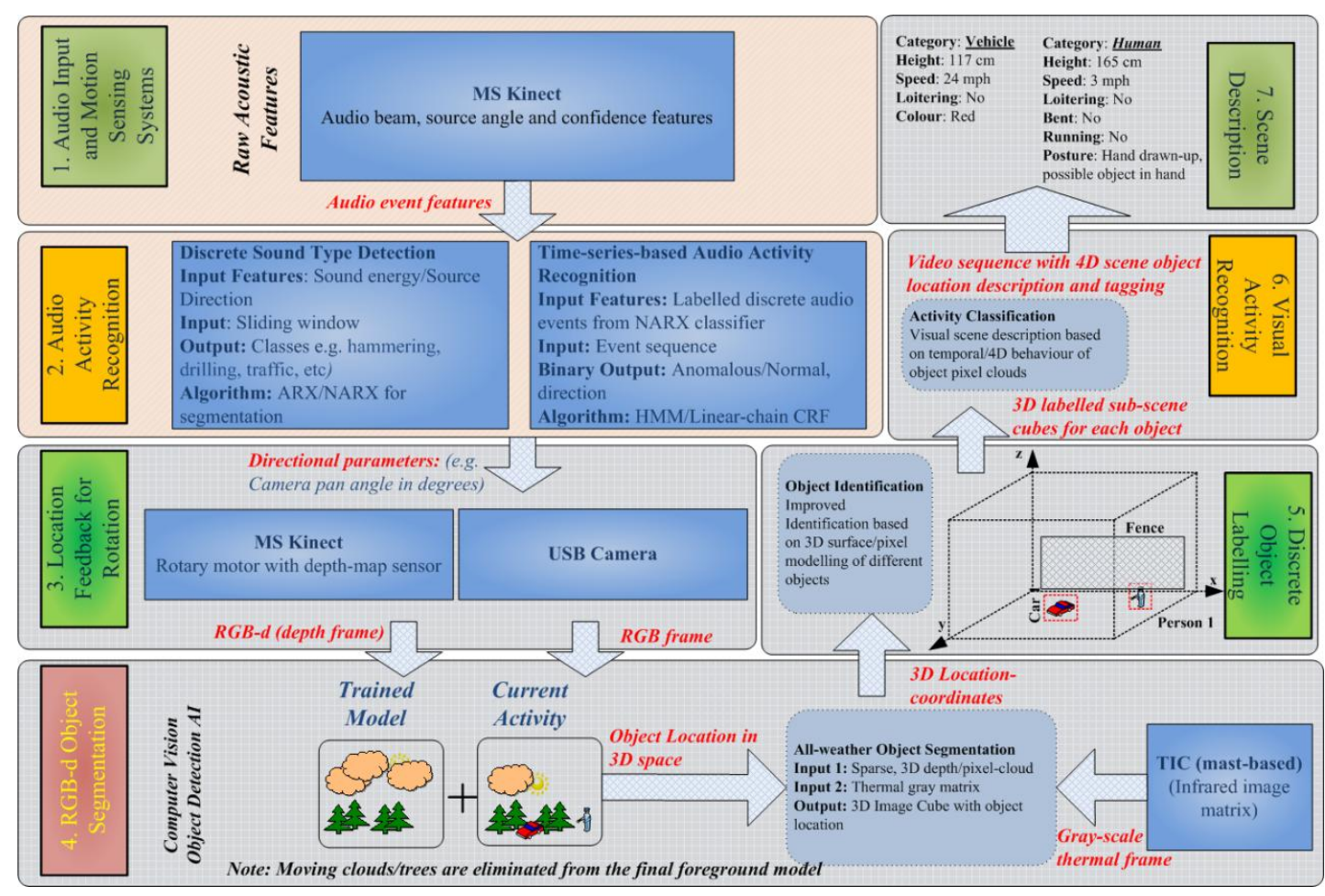

Figure 3: Proposed system architecture of a multi-sensor, 4D smart perimeter surveillance system with the scope of this research shown in shaded cells under (1) Audio Input and Motion Sensing System and (2) Audio Activity Recognition 


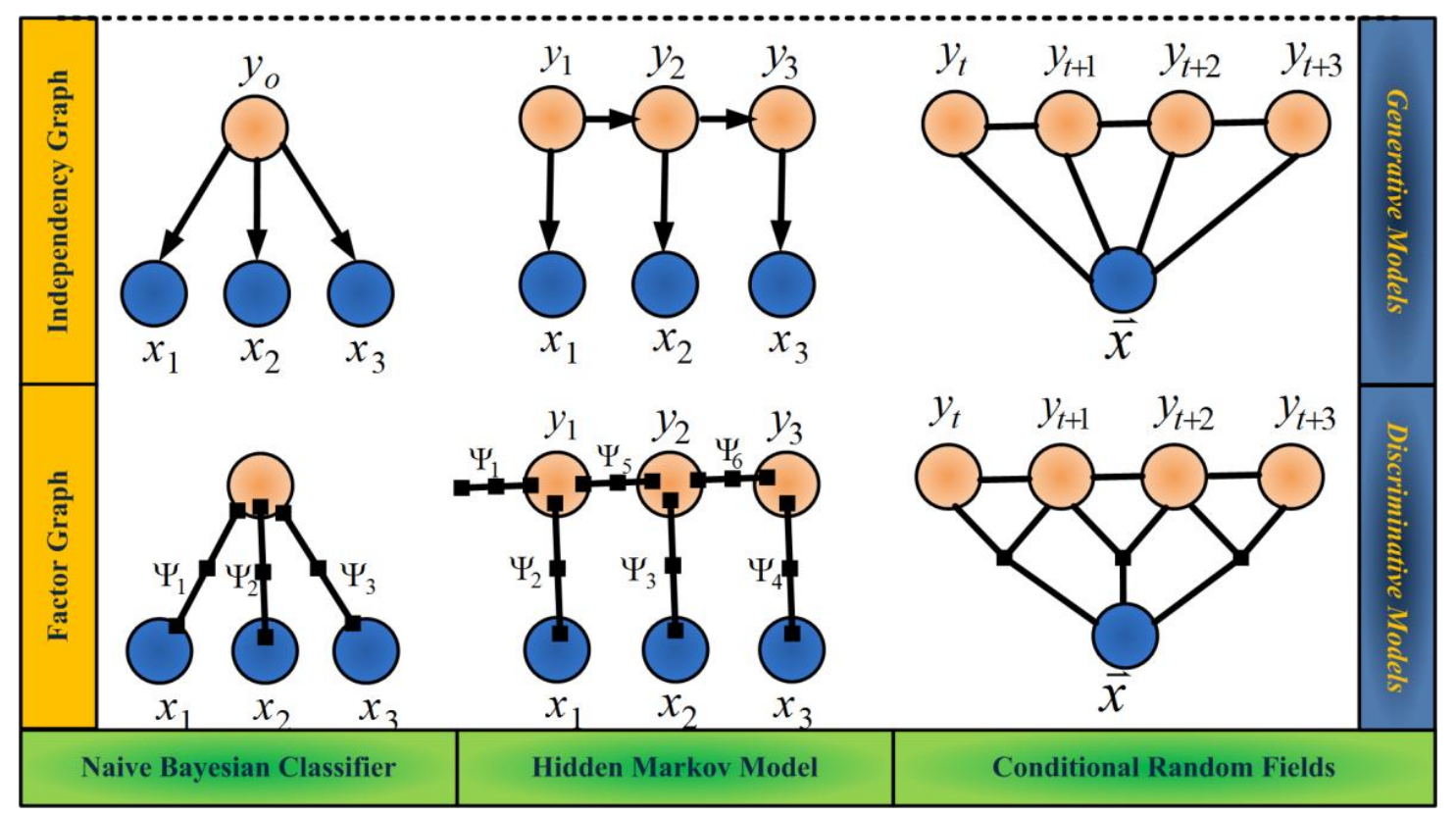

Figure 4: Transformation from discrete to time-series sequence HMM and the ultimate linear chain CRFs representation

\subsection{Directional sound segmentation via non-linear autoregressive modeling}

The first stage of the single-channel classification model utilized a nonlinear autoregressive forecasting model with exogenous input (NARX) to predict the start and end of specific sound activity segments in the presence of background noise [17]. In this so-called NARX model structure, a soundevent at a given time is described by a measure of sound energy during the previous time instances based on the exogenous direction variable to track time-specific patterns and energy variations. An example situation for the $9-10$ p.m. time instance in an office environment can predominantly contain sound data where the majority of sound energy activity is focused in the direction where the door is located which is used by staff members to report for work.

This time and energy-driven technique is deemed effective in real-world-applications where certain audio events only take place during specific time instances and in particular directions. MFCC ((MelFrequency Cepstral Coefficients), PLP (Perceptual Linear Prediction), LPC (Linear Predictive Coding), ZCR (Zero Crossing Rate) and pitch frequency are a few sound feature extraction procedures as reported by Portelo et al. [18] and Atal \& Remde [19].

In order to keep track of a daily, hourly pattern, a binary-valued vector $\mathcal{H}_{t} \in\{0,1\}^{24}$ is used with a 1 for each hour of the day of the "pattern window" being observed at time $t$ where, feature vector for 9 p.m. is represented by a vector $[0,0,0,0,0,0,0,0,1,0,0,0,0,0,0,0,0,0,0,0,0,0,0,0$, ] for a directional sound observed at that time (shown in Figure 9). The energy variable $\varepsilon_{i}$ is the observed sound energy at a time instance $t$ defined in seconds for the floor plan shown in Figure 9. The variable $\varepsilon_{i} b$ is generated as a fractional value between $0-1$ which is simplified as a binary-valued vector $\varepsilon_{t} \epsilon\{0,1\}^{10}$ with each value representing a range of 0.1 energy intervals.

The underlying NARX model thus contains the following autoregressive and exogenous variables (Figure 5):

1. An autoregressive set of 10 : 
a. Previous energy variables represented by $\varepsilon_{t} \epsilon\{0,1\}^{10}$ for a 5 -second-lag window and containing energy summation of MFCC, LPC and delta LPC values. [18]

b. Confidence-related variables $\mathcal{C}_{t} \in\{0,1\}^{10}$ to further improve the directional confidence, and

c. Hourly time information based on 24 variables for each hour of the day represented by $\mathcal{H}_{t} \in\{0,1\}^{24}$

2. Direction-related exogenous variables $\mathcal{D}_{t} \in\{0,1\}^{10}$ with each representing 10-degrees from left-toright for the entire 5 -second-window thereby representing a total of 100 degrees

Therefore, for each regression vector sample $v_{t}$, a total of $10+10+24+10=54$ variables are to be included for NARX training as follows:

$x_{\mathrm{t}}=f\left(v_{\mathrm{t}}\right)+\mathrm{err}$

In the equation above, $f$ is the unknown function, $x_{\mathrm{t}}$ is the classification output (i.e. normal/anomalous activity), err is the error term and $v_{t} \square \mathbb{R}^{n}$ is the feature vector for NARX training further defined as

$v_{\mathrm{t}}=\left[x_{t-1}, \ldots, x_{t-10}, \mathcal{E}_{t}, \mathcal{C}_{t}, \mathcal{H}_{t}, \mathcal{D}_{t}\right]$

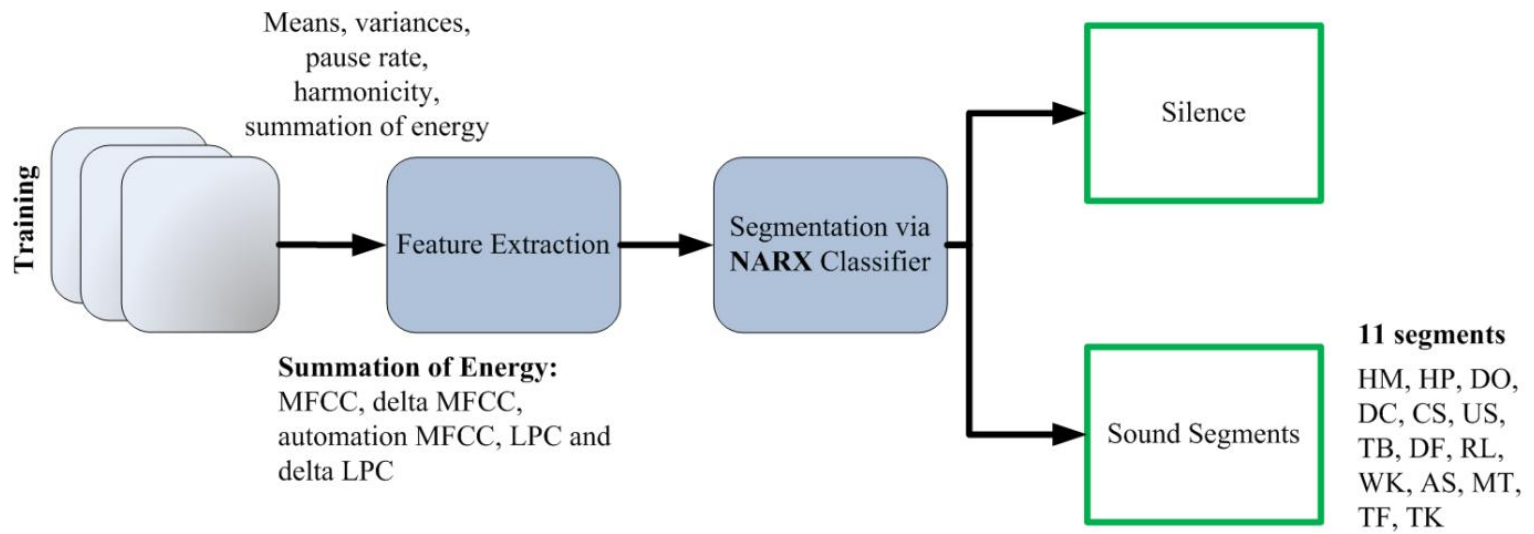

Figure 5: Block diagram of the classification of non-silent activity segments via an autoregressive neural classifier (NARX) with directional audio as exogenous data. The Summation of Energy features are actively utilized in sound synthesis research as reported by Portelo et al. [22]

\subsection{A HMM/CRF-based audio event detection system}

Real-world systems are defined not over individual and discrete signatures and are generally based on the assessment of time-series-based, inter-connected events. The case is true for audio/visual behavioral profiling systems where a set number of temporally sequential events are modeled to depict specific conditions [20]. Such temporally-defined signals are modeled via intrinsically nonlinear Bayesian Belief Networks or HMMs that are capable of parsing time-based sequence information into the most closely identified state [20,21]. For instance, a specific card-based door scan entry system into a secure building is primarily based upon a number of discrete events connected together. Figure 6 demonstrates a particular case where a person scans a staff card to gain entry into an office space. A typically valid process in such a case is marked with a successful, single bleep sound, door opening sound, walking steps and a door closing sound. A number of these sound 
signatures may overlap and there may be variable-length-delays between various activities. Nonetheless, a multi-state case similar to this can be defined via a temporally heuristic or statistical process.

\subsubsection{The HMM/CRF classification model}

A linear-chain CRF construction from the case shown in Figure 8 describes potential state transition conditions or acoustic state transitions during a secure door entry situation. The first step to classify a sequence derived from this situation is to keep the variables/sequence of states $y$ hidden and obtain a model called the Hidden Conditional Random Field. Analogous to the HMM, CRFs have several potential factors associated with each class variable. Each potential function has a group of weights and features with each element in the weight array associated to an element in the feature set.

Based on each of the discrete events shown in Figure 6, a particular real-world scene can be hierarchically defined to contain a set of acoustic events with each containing a number of sub acoustic events with each contain different sound signatures. In order to further clarify the process, a card-based secure door entry system is shown in Figure 6. A normal card-based operation comprise of a user scanner his or her card on a door scanner where a successful scan grants an entry. An entry event can further generate a door opening sound, a footsteps sound and a door closing sound. In order to train such a model, positive data sample may comprise of a wide range of options in which each or a number of sub-events mentioned above are repeated in different ways. For instance, a user may unsuccessfully scan his/her card a set number of times due to some reason before success. An unsuccessful attempt will generate a recurring bleep sound compared to a single bleep heard when a successful scan is made. Moreover, door closing sound may not be heard for extended periods reaching up to a length of 30 seconds if the user is talking while standing on the door thereby inducing an additional talking profile. Finally, based on the type of footwear, the sound of footsteps may never be heard for a person at all. 
Hierarchical sound profile segmentation

from a pre-labelled set of models

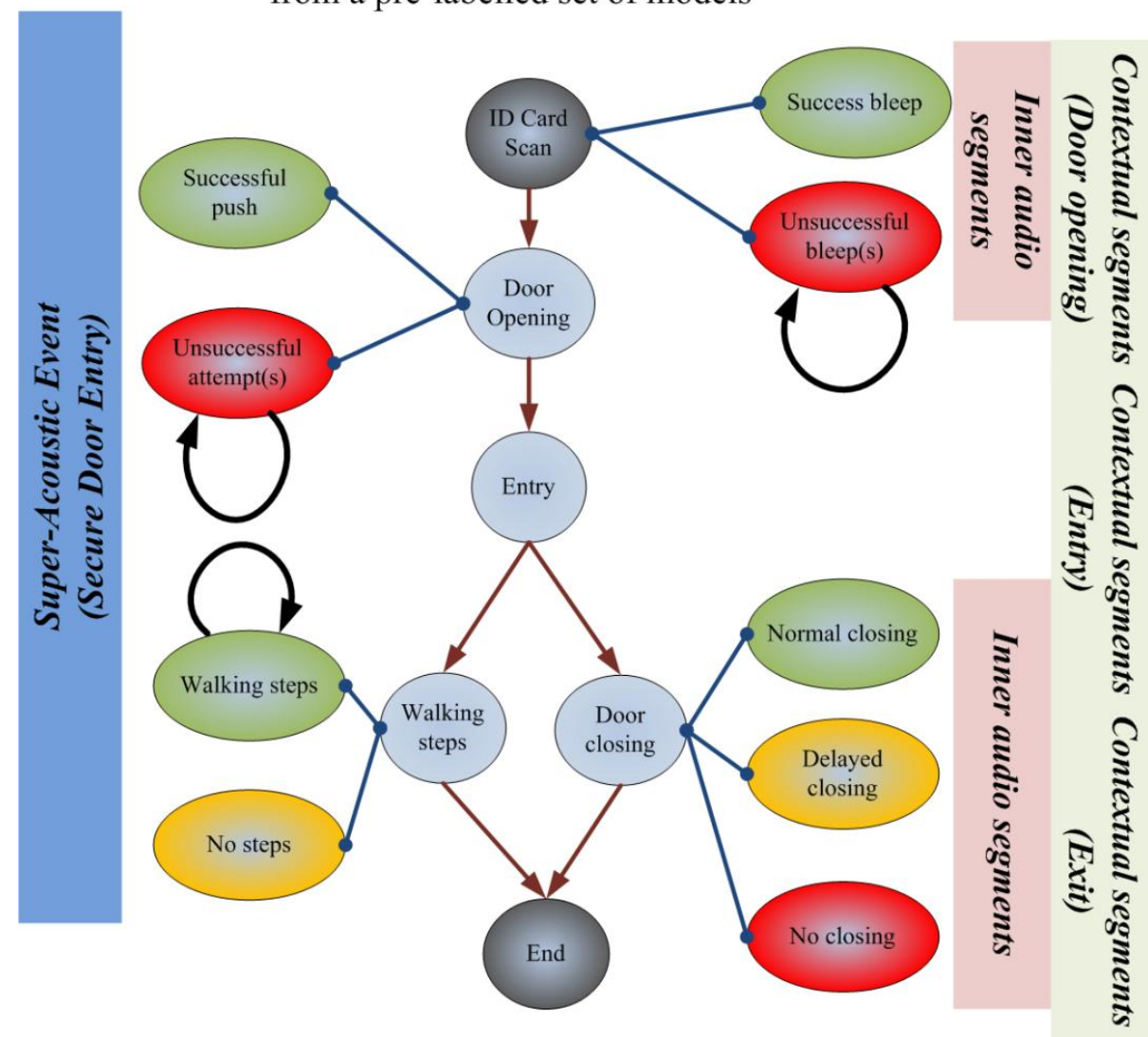

Figure 6: State transition sequence for the proposed HMM/CRF system

$\begin{array}{cccc}\text { Card scan } & \text { Latch operation with door } & \text { Door } & \text { First step of } \\ \text { bleep } & \text { opening } & \text { closing } & n \text { steps }\end{array}$

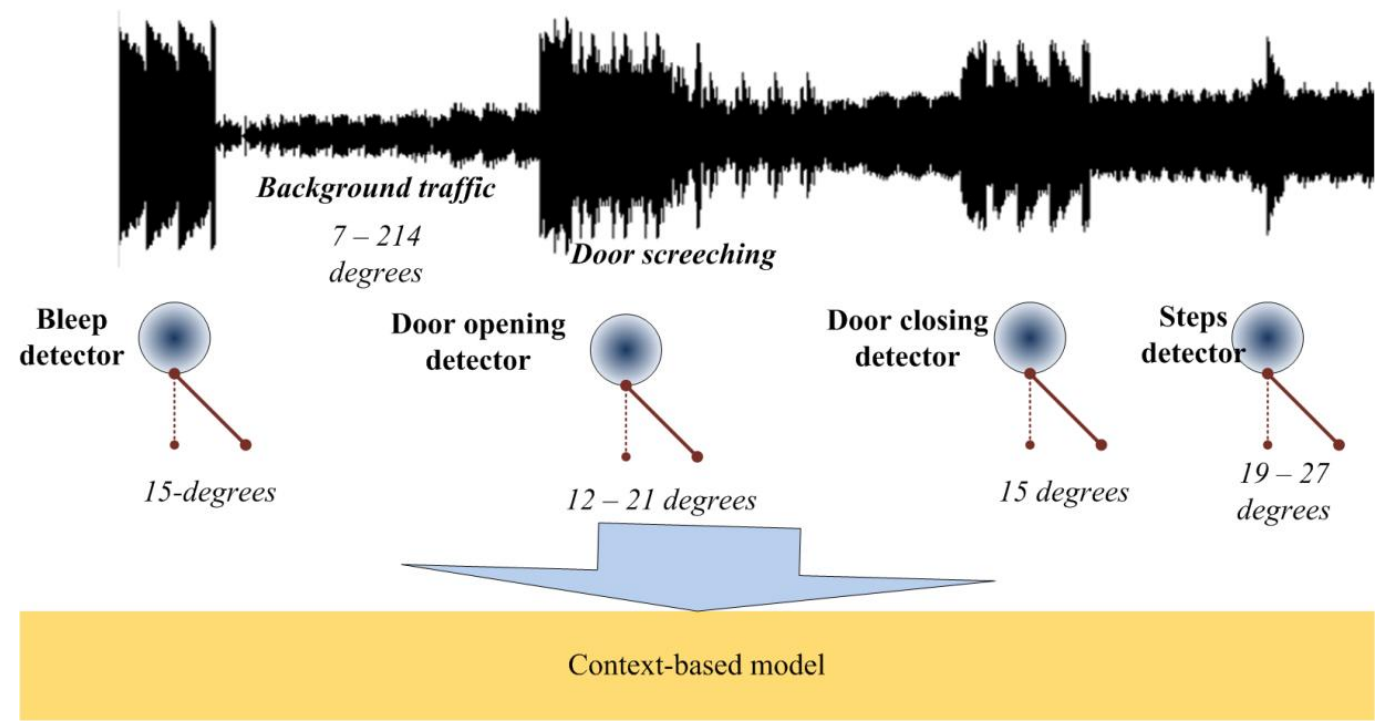

Figure 7: Modeling of linear chain CRF into context-based individual models

The CRF/HMM data parsing, training and testing algorithms based on Baum-Welch learning are shown in Table 1. The system is trained over a multivariate normal distribution of data comprising of 
the direction and energy audio features captured via a MS Kinect device/SDK. As the situation might be extended to more-than-two variables currently used as an input feature set, the distribution shall be regulated via two parameters, the mean vector and the variance-covariance matrix.

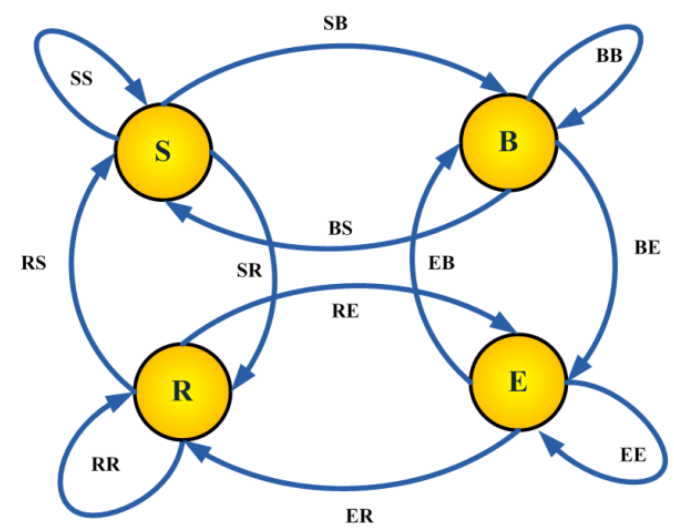

Acoustic States

B Card Bleep (CS); E: Door Entry (DO); S: Step Sound (WK);

R: Door Release (DC)

Figure 8: A stochastic finite state automaton for a door-entry-sound-sequence case

It must be noted that the algorithm is using a fixed number of states (4) which is based on some "apriori" knowledge of the system which gave the lowest error margins for both $\mathcal{C}_{\mathrm{HMM}} / \mathcal{C}_{\mathrm{CRF}}$ classifiers. As the CRF is a generalization of the HMM, any combination of discrete or continuous feature functions can be used in modeling. Once a HMM is learned, its final probabilities are used to initialize the subsequent CRFs instead of starting from random/guessed values. As the values are initialized on the basis of a continuous density HMM, all the feature functions directly operate over the observed $\mathrm{Y}_{\mathrm{i}}=\lambda_{\mathrm{i}}: \varepsilon_{\mathrm{i}}$ samples for a time sequence $\mathrm{T}_{\mathrm{i}}$ where $0 \leq \mathrm{i} \leq \mathrm{N}$ and $\mathrm{N}$ is the number of feature-pairs $\lambda_{\mathrm{i}}: \varepsilon_{\mathrm{i}}$ in an audio event.

Table 1: Data parsing, training and testing algorithm for HMM/CRF with the acoustic data repository

Kinect Direction Audio/Energy Capture Algorithm

HMM Data Parsing:

Begin:

For audio sequence $\mathrm{T}_{i} 0 \leq i \leq N$

Get Kinect directional audio/audio energy

Construct feature vector $Y_{i}=\lambda_{i}: \varepsilon_{i}$ where $\lambda$ is the audio angle and $\varepsilon$ is the audio energy

For Each vector $Y$

Return ZScores $\mathbb{Z}_{i}$

Add $\mathbb{Z}_{i}$ and class label $\Phi_{i}$ to training database $\mathfrak{D}$

HMM Training (BaumWelch Learning):

Define $H M M$ classifier $\mathcal{C}_{H M M}$ with a multivariate normal distribution

Set Convergence Criteria States: 5; Iterations 0; Tolerance 0.01 and Rejection to False

Calculate HMM Classification Error $e_{H M M}$

For CRF classifier $\mathcal{C}_{C R F}$ use Resilient Back Propagation learning classifier $\mathcal{C}$

Set Convergence Criteria Iterations 100; Tolerance 0.01

Calculate CRF Classification Error $e_{C R F}$ 
Testing

For Each feature sample Y

Identify classification categories with $\mathcal{C}_{H M M} / \mathcal{C}_{C R F}$

End

\section{Experimental Results}

The tests were carried-out in a semi-open-plan office environment of a first-floor, newly refurbished academic building with windowed walls on the entire three out of four sides of the floor. All of these three walls opened to busy roads with a 24-hour continuous traffic flow (Figure 9). All the rooms were equipped with soundproofing measures. The personalized set of data was collected via MS Kinect device using Kinect SDK with software developed via Visual Studio 2012/2013 and .Net Framework 4.5. The previously-shown Figure 1(b) represents Kinect's depth and skeleton data (not addressed/utilized in the current research context) and the directional audio feature capturing interface was implemented via Kinect SDK's inbuilt beam-forming, triangulation and confidence-level assessment algorithms. It must be noted that, since the personalized dataset contained directional sound data as well, the AL-CMU datasets were re-recorded with the same configuration while placing the speakers as live sound sources. The positions of these speakers at various angles are shown as colored circles in Figure 9. The figure also represents the estimated position of sound-direction-areas or envelopes from where the majority of respective sound signatures originated. For instance, traffic sound signatures primarily came from the road-side windows (Pink envelope) whereas most of footstep sounds came from the lobby and hallway area (Green envelope) and high-confidence doorentry signatures originated from the Main Entry Direction shown as a blue envelope.

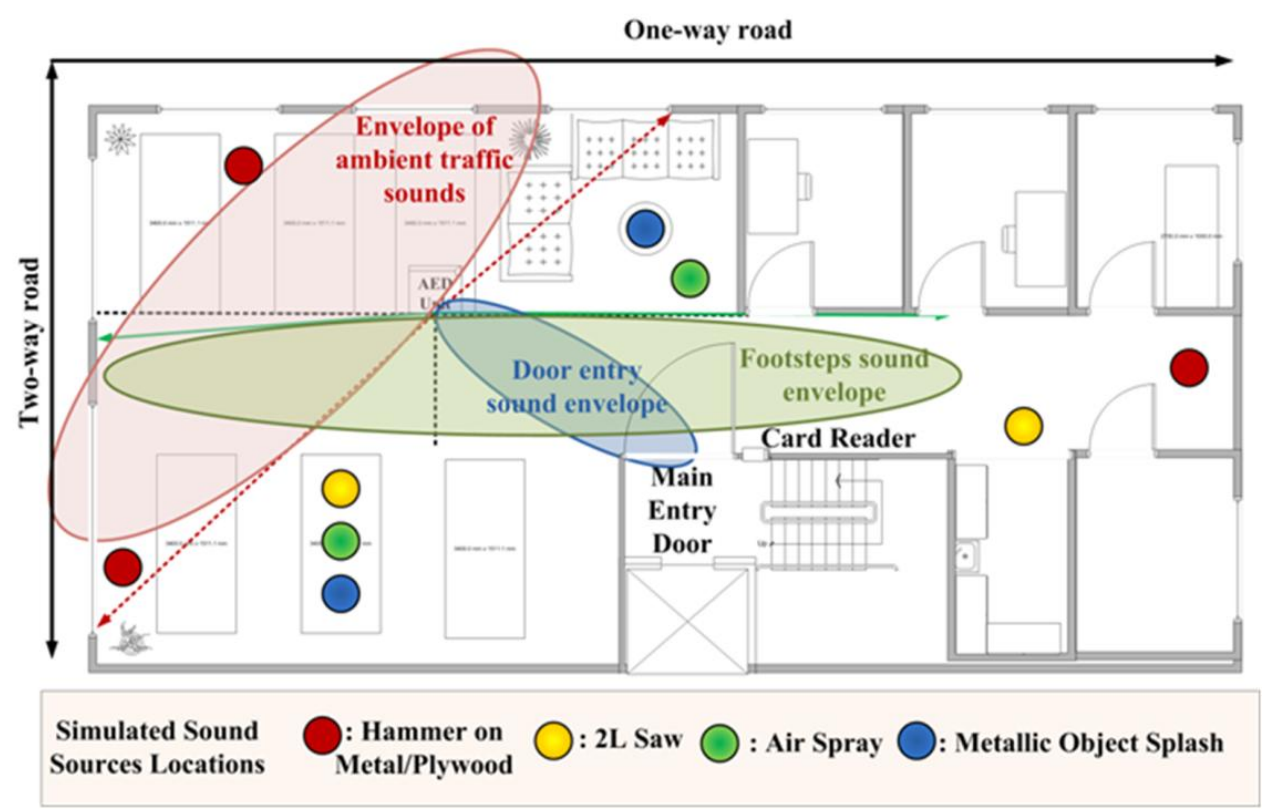

Figure 9: Floor plan of an office environment for indoor audio-event-detection and identification experiment with microphone array position (MS Kinect-based AED Unit), likely sound envelopes of various sound activities and positions of various simulated sound sources 


\subsection{Quad-channel segmentation results}

The study gathered a mix of datasets from the Auditory Lab, Carnegie Melon University (AL-CMU) (Column 2, Table 2) AL-CMU data was originally recorded at $16-b i t, 44.1 \mathrm{kHz}$ recorded via a matched pair of Earthworks QTC30 microphones, USBPre 1.1 microphone amplifier and an analogue to digital PC interface. The recordings were made via Audacity open-source recording software and the microphones kept $20 \mathrm{~cm}$ apart. For this research the entire Auditory Lab and personally recorded audio event data was captured via MS Kinect-based configuration shown in Figure 1(b). The events were captured at 16-bit, $44.1 \mathrm{kHz}$ and also incorporated three directional acoustic features of Audio Beam, Source Angle and Source Confidence.

Figure 10 shows the results of an indoor sound recording session representing regular multidirectional sound sources with (a) showing muffled speech in an office environment with at least 4 group-talkers located on the right-hand-side of the sensor whereas (b) depicting a hammering sound $(1-2.5 \mathrm{~s})$ and a door-closing sound (8.5-9.5s) at a direction of 0-degrees (See arrow direction in (b)).

The Audio Beam property by MS Kinect captures the directional audio cone of a sound source to improve its overall distance perception. The Source Angle identifies the direction of a sound source from +50 to -50 degree angles. A positive value indicates sound source located on the left side of the sensor and vice versa whereas a 0 indicates a centrally-located sound source. These values were normalized to range from 0 to 100 with the angle 50 representing middle location for training purposes. The Source Confidence describes the confidence level with which a specific sound source is located. It must be noted that in a communal talking environment, muffled speech of multiple talkers standing close to each other will have a lower Source Confidence level compared to a door closing sound. In Figure 10 (b), a higher confidence level of 0.54 is seen compared to 0.32 in (a) based on rate-of-confidence with which a sound source is located. The aspect provides crucial information for audio events in the presence of ambient noise and thereby assists in the segmentation of particular segments-of-interests for the subsequent HMM training.

\subsection{Empirical evaluation of audio event detection via autoregressive modeling}

Based on the NARX/ARX models trained as explained in the previous section, the first phase evaluated individual audio events as described in Table 2. A total of 460 audio samples, recorded as explained in Section 0, were used with a total duration of 105 seconds with and without exogenous values.

Table 3 shows the lowest identification accuracies achieved for deformation (DF), traffic (TF) and door closing (DC) categories for ARX classification without exogenous input. These lower accuracies may be attributed to the predominantly monotonous or repetitive nature of the feature set which is often similar in energy with each other.

Results from the second (NARX) model contained exogenous directional sound attribute $\mathcal{D}$ aimed at eliminating background noise from random directions based on the presumption that background noise matching the direction of a genuine audio event had a very low likelihood (Table 4). The outcome represents a substantial improvement in event detection accuracy by the introduction of the exogenous data. The lowest reported accuracies shown in Table 4 were mainly from deformation activity chainsaw (DF), traffic (TF), and door closing (AS) events. 


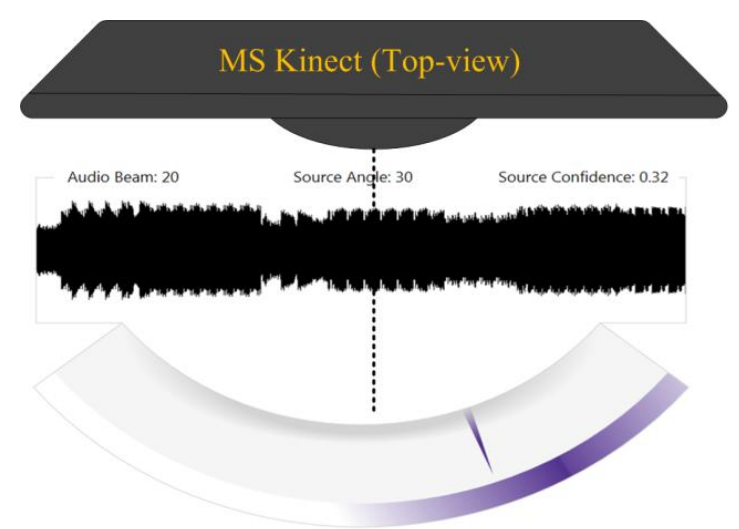

Regular office noise detected at 27/11/2013 09:02:54

(a)

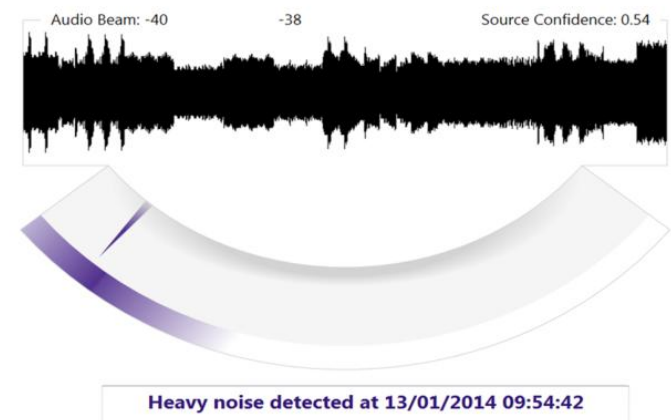

(b)

Figure 10: MS Kinect configuration (via MS Kinect SDK) for energy/directional sound recording of events including (a) regular office conversation (TK) on the right-hand-side and (b) hammering (HM) on the left-hand-side with a high confidence level

Table 2: List of audio events recorded from the AL-CMU database and locally-extract audio data to train a Naïve Bayesian classifier for discrete audio event classification

\begin{tabular}{|l|l|l|}
\hline Sound Events & $\begin{array}{l}\text { AL-CMU (Class } \\
\text { Representation Code) }\end{array}$ & $\begin{array}{l}\text { Local dataset } \\
\text { Representation Code) }\end{array}$ \\
\hline Impact & $\begin{array}{l}\text { Hammer on Metal (HM), } \\
\text { Hammer on Plywood (HP) }\end{array}$ & $\begin{array}{l}\text { Door Opening (DO), } \\
\text { Door Closing (DC) }\end{array}$ \\
\hline Electronic & - & $\begin{array}{l}\text { Successful Card Bleep (CS), } \\
\text { Unsuccessful Card Bleep (US), } \\
\text { Telephone Bleep (TB) }\end{array}$ \\
\hline Deformation & $\begin{array}{l}\text { Sawing via 2-L Japanese Saw } \\
(\mathbf{D F})\end{array}$ & - \\
\hline Rolling & Rolling Marble on Metal (RL) & Walking (WK) \\
\hline Air & Air Spray Can (AS) & - \\
\hline Water & Metal Splash (MT) & - \\
\hline $\begin{array}{l}\text { Background } \\
\text { Noise }\end{array}$ & - & Traffic (TF); Talking (TK) \\
\hline
\end{tabular}

Table 3: Auto-regression (ARX) classification without exogenous data via feature set extracted over 5-second-windows 


\begin{tabular}{|c|c|c|c|c|c|c|}
\hline $\begin{array}{l}\text { Event } \\
\text { Type }\end{array}$ & $\begin{array}{c}\text { \# of } \\
\text { Samples } \\
\text { Tested }\end{array}$ & $\begin{array}{c}\text { Average } \\
\text { Sub- } \\
\text { event } \\
\text { Length } \\
\text { (seconds) }\end{array}$ & $\begin{array}{l}\text { Complete } \\
\text { Event } \\
\text { Lengths } \\
\text { (seconds) }\end{array}$ & $\begin{array}{l}\text { Samples } \\
\text { Incorrectly } \\
\text { Classified }\end{array}$ & $\begin{array}{c}\text { Classification } \\
\text { Accuracy } \\
(\%)\end{array}$ & $\begin{array}{l}\text { Duration } \\
\text { (seconds) }\end{array}$ \\
\hline $\mathrm{HM}$ & 40 & 3.4 & 10 & 7 & 82.5 & 136 \\
\hline HP & 40 & 3.9 & 10 & 5 & 87.5 & 156 \\
\hline $\mathrm{DF}$ & 20 & 2.3 & 10 & 6 & 70.0 & 46 \\
\hline RL & 20 & 2.2 & 7 & 2 & 90.0 & 44 \\
\hline $\mathrm{AS}$ & 20 & 1.9 & 7 & 4 & 80.0 & 38 \\
\hline MT & 20 & 0.7 & 7 & 1 & 95.0 & 14 \\
\hline $\mathrm{DO}$ & 60 & 1.3 & 10 & 1 & 98.3 & 78 \\
\hline $\mathrm{DC}$ & 60 & 1.4 & 10 & 13 & 78.3 & 84 \\
\hline $\mathrm{CS}$ & 60 & 0.7 & 7 & 1 & 98.3 & 42 \\
\hline WK & 40 & 0.7 & 7 & 7 & 82.5 & 28 \\
\hline TK & 40 & 2 & 10 & 5 & 87.5 & 80 \\
\hline \multirow[t]{2}{*}{$\mathrm{TF}$} & 40 & 2 & 10 & 11 & 72.5 & 80 \\
\hline & 460 & 22.5 & 105 & 63 & 85.2 & 826 \\
\hline
\end{tabular}

Table 4: Auto-regression (NARX) classification with exogenous data via feature set extracted over 5-second-windows with audio direction $\mathcal{D}$ as the exogenous variable

\begin{tabular}{|c|c|c|c|c|c|c|}
\hline $\begin{array}{c}\text { Event } \\
\text { Type }\end{array}$ & $\begin{array}{c}\text { \# of } \\
\text { Samples } \\
\text { Tested }\end{array}$ & $\begin{array}{c}\text { Average } \\
\text { Sub-event } \\
\text { Length } \\
\text { (seconds) }\end{array}$ & $\begin{array}{c}\text { Complete } \\
\text { Event } \\
\text { Lengths } \\
\text { (seconds) }\end{array}$ & $\begin{array}{c}\text { Samples } \\
\text { Incorrectly } \\
\text { Classified }\end{array}$ & $\begin{array}{c}\text { Classification } \\
\text { Accuracy } \\
(\%)\end{array}$ & $\begin{array}{c}\text { Duration } \\
\text { (seconds) }\end{array}$ \\
\hline HM & 40 & 3.4 & 10 & 3 & 92.5 & 136 \\
\hline HP & 40 & 3.9 & 10 & 2 & 95.0 & 156 \\
\hline DF & 20 & 2.3 & 10 & 4 & 80.0 & 46 \\
\hline RL & 20 & 2.2 & 7 & 2 & 90.0 & 44 \\
\hline AS & 20 & 1.9 & 7 & 3 & 85.0 & 38 \\
\hline MT & 20 & 0.7 & 7 & 0 & 100.0 & 14 \\
\hline DO & 60 & 1.3 & 10 & 1 & 98.3 & 78 \\
\hline DC & 60 & 1.4 & 10 & 7 & 88.3 & 84 \\
\hline CS & 60 & 0.7 & 7 & 0 & 100.0 & 42 \\
\hline WK & 40 & 0.7 & 7 & 3 & 92.5 & 28 \\
\hline TK & 40 & 2 & 10 & 3 & 92.5 & 80 \\
\hline TF & 40 & 2 & 10 & 7 & 82.5 & 80 \\
\hline & 460 & 22.5 & 105 & 35 & 91.4 & 826 \\
\hline
\end{tabular}

The measure of miss-classification for both the models is further shown in Figure 11 where the midsection of the triangular cones represents the number of incorrect classifications of a specific category. For instance, in Figure 11 (a),

- Four instances of Hammer-on-Metal (HM) are shown to be miss-classified Hammer-on-Plywood (HP) and three instances miss-classified as Walking (WK) (Table 3). 
- This ARX-based outcome from Table 3 is shown to improve in Figure 11 (b) with the introduction of the exogenous variable where only three instances of $\mathrm{HM}$ are miss-classified as HP (Table 4)

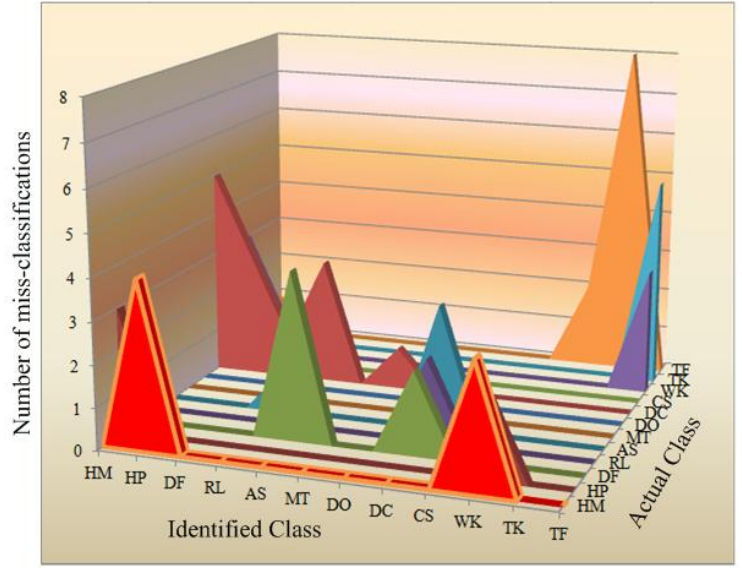

(a)

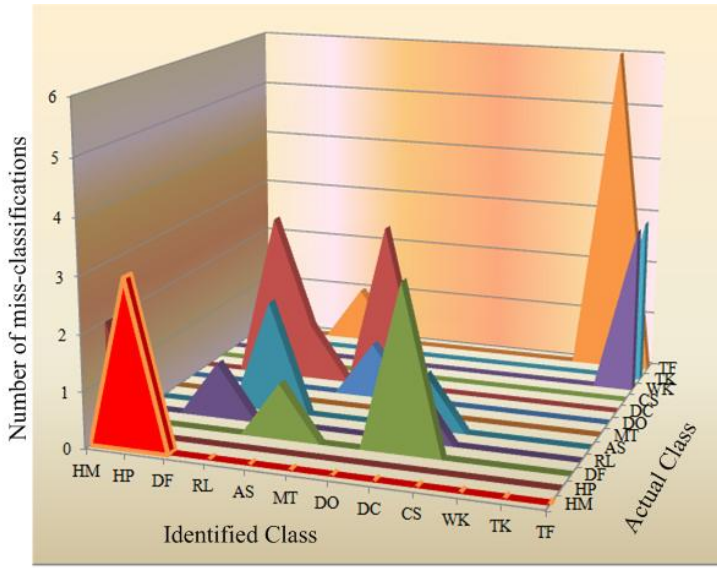

(b)

Figure 11: Miss-classification matrix based on the autoregressive classification without exogenous input in (a) and with exogenous data in (b) showing marked improvement in segmentation accuracy.

\subsection{Evaluation of audio events via HMM/CRF}

The previous section demonstrates identification outcome of discretely segmented audio events based on direction data. This section presents the classification accuracy of the identified, time-chained events recorded over a temporal scale. Figure 12 demonstrates a sparse-matrix of state-transition based on sound angle/energy plot on a temporal scale (a) Office Talk (TK) (b) Foot-steps (WK), (c) Hammer-on-Plywood (HP) and (d) Door opening (DO). As discussed above, the feature vector of these audio signatures comprised of a multivariate signature containing three audio signals of signal energy, source angle, and source confidence.

The labeled segments from the previous NARX model were manually labeled to prevent identification inaccuracies from the previous section from creeping in the time-series classification model described in this section. The temporal context exploited the periodic nature of the majority of events in the indoor test setting which mainly comprised of muffled speech along with occasional room entries via a card-based system. Based on the NARX/manual labeling via phase one, a set of normal and abnormal behaviors identified as follows (Table 5) 
Table 5: State sequence samples representing various indoor audio behaviors evaluated with the HMM/CRF classifiers (Legend of states described in Table 2)

\begin{tabular}{|l|l|l|}
\hline $\begin{array}{l}\text { Output } \\
\text { Class }\end{array}$ & State Sequence Sample & Remarks \\
\hline Normal & CS->DO->WK->DC & $\begin{array}{l}\text { A person continues on walking after a } \\
\text { successful card scan }\end{array}$ \\
\hline Normal & CS->DO->DC->WK & $\begin{array}{l}\text { A person pausing for a while having } \\
\text { entered the room thereby allowing the } \\
\text { door to close first before walking away }\end{array}$ \\
\hline Anomalous & US->US (repetitive) & $\begin{array}{l}\text { Repeated unsuccessful card scan } \\
\text { attempts }\end{array}$ \\
\hline Anomalous & DO->WK->DC; DO->DC->WK & Entry without card scan \\
\hline Anomalous & US->DO->WK->DC; US->DO->DC->WK & Possible case of improperly shut door \\
\hline
\end{tabular}

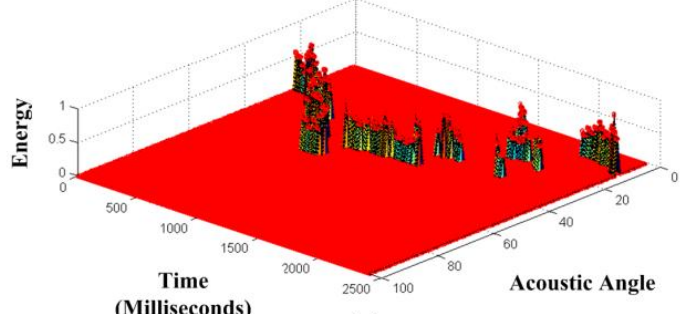

(a)

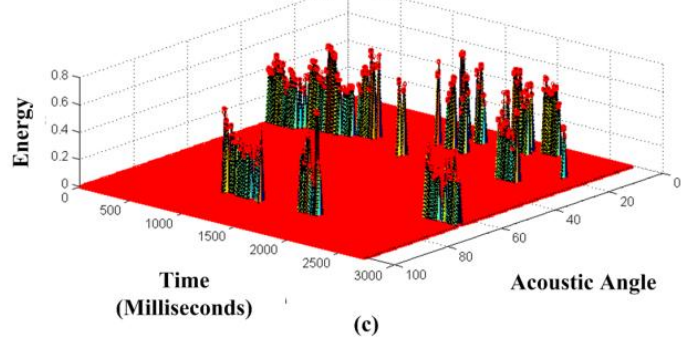

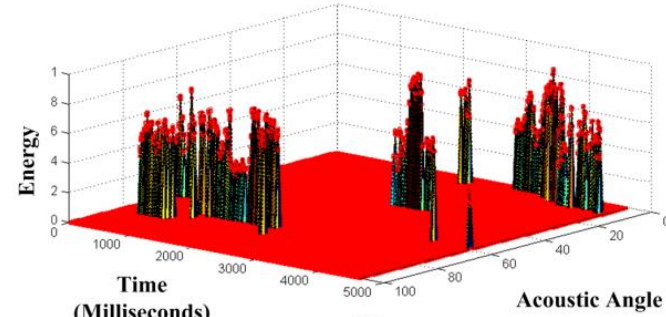

(b)

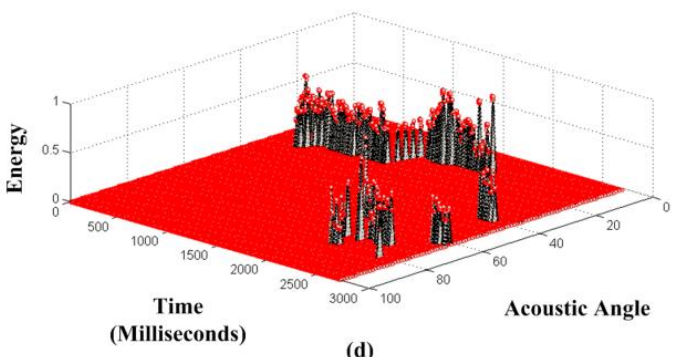

Figure 12: A sparse-matrix of state-transition based on sound angle/energy plot on a temporal scale (a) Office Talk (TK) (b) Foot-steps (WK), (c) Hammer-on-Plywood (HP) and (d) Door opening (DO)

\subsubsection{HMM/CRF training and evaluation}

For each case described in Table 5, the labels given by the HMM/CRF system are compared to the annotated/manually labeled ground-truth audio sequences for the specific event. The overall performance is computed by calculating a false sub-event rejection ratio (FR) against a false detection ratio (FD) defined as follows:

$$
\begin{aligned}
& \text { FSR }=\frac{\# \text { of failed sub }- \text { event classification }}{\# \text { of total sub }- \text { events to classify }} \\
& \text { FDR }=\frac{\# \text { of incorrectly identified activities }}{\# \text { of total recorded audio sample }}
\end{aligned}
$$

The training data for this evaluation was based on audio clips/samples recorded in the presence of normal background noise including (1) traffic and (2) usual outdoor events and also in (3) absolute silence. The experiments are further explained in the next sections.

The initial experiment was aimed to understand the impact of background noise on the sub-event and audio sample cases. For both the cases, a total of 110 clips were recorded to evaluate the trained 
HMM/CRF algorithms in the presence of noise as well as a stable dataset. Noise in data in this case is described as the regular office noise, road traffic sounds and other urban audio signatures happening during the process of recording. The complete event failure (CEF) rate is the last column shown in Table 6. A failure may occur if, due to one or more sub-event failures (SEFs), the underlying Activity is not correctly classified. It may be a case that despite a SEF, the state-sequence recognized still classifies the event as a valid Normal or Anomalous class. For instance, for the CS->DO->DC->WK, the event can still be recognized as a valid Normal event even if the last WK sub-event is not correctly identified. On the other hand, the US->DO->WK->DC Activity shall still be classified as an Anomalous class despite its last two sub-events not being correctly classified (WK->DC).

Table 8 represents a better CEF rate with the NARX/CRF model with a complete event failure CEF rate of $19.09 \%$ which demonstrates the superiority of CRF on HMM for time-series audio event recognition.

Table 6 reports a summary of sub-event recognition failures of noisy and stable data when evaluated against models defined as follows:

\subsubsection{NARX/HMM model with noisy/stable data:}

- NARX-based individual event labeling algorithm corrected and labeled manually

- HMM trained over multivariate Gaussian distribution via a Baum-Welch learning algorithm

\subsubsection{NARX/CRF model with noisy/stable data:}

- NARX-based individual event labeling algorithm corrected and labeled manually

- Training with CRF via a Hidden Resilient Gradient Learning algorithm

It must be noted that the CRF model uses a Resilient Back Propagation algorithm based upon its best performance against the task at hand. However, other algorithms such as the Stochastic Gradient Descent can be used as well. The Hidden CRF used in this work is a generalization of a HMM classifier where any discrete, continuous or a mixture of function features can be used.

The model performance in Table 6 shows sub-event recognition failure rates for the two models described above. Total event recognition (TER) for both the noisy and stable data was recorded in 110 clips. These clips comprised of a number of sub-event categories (SEC) which can be checked with the Activity column as well. The failure rate is shown in two different respects and columns represented as the sub-event failure (SEF) and the complete event recognition failure (CEF) where, the CEF value is not a cumulative sum of SEF due to the fact that more-than-one SEF may occur in a single audio event. SEF was particularly higher in noisy data for the four main categories of CS, DO, WK and DC (Table 2). The blacked-out cells show N/A sub-events for each activity.

\subsubsection{Sub-event accuracies}

This SEC failure data can further be used to cross-evaluate the NARX audio event outcomes shown in the previous section (Table 4). For instance, the activities of CS->DO->WK->DC and CS->DO-> DC -> WK given individual accuracies of $100 \%, 98.3 \%, 88.3 \%$ and $92.5 \%$ for CS, DO, DC, and WK respectively in Table 4. The SECs for the four categories in Table 6 (NARX/CRF Stable Data) represent $0,2,6$ and 0 failures for CS, DO, DC, and WK with 25 SECs each thereby given a success rate of $100 \%, 92 \%, 80 \%, 76 \%$ and $100 \%$.

\subsubsection{Overall event recognition rate}

The complete event failure (CEF) rate is the last column shown in Table 6. A failure may occur if, due to one or more sub-event failures (SEFs), the underlying Activity is not correctly classified. It may be 
a case that despite a SEF, the state-sequence recognized still classifies the event as a valid Normal or Anomalous class. For instance, for the CS->DO->DC->WK, the event can still be recognized as a valid Normal event even if the last "WK sub-event is not correctly identified. On the other hand, the US->DO->WK->DC Activity shall still be classified as an Anomalous class despite its last two subevents not being correctly classified (WK->DC).

Table 7 represents a better CEF rate with the NARX/CRF model with a complete event failure CEF rate of $19.09 \%$ which demonstrates the superiority of CRF on HMM for time-series audio event recognition.

\subsubsection{Benchmarking with previous work}

Audio event detection and segmentation has frequently been addressed in the recent literature. Portelo et al. [18] evaluated single channel sound data with PLP or MFCC features with SVM and HMMbased classifiers. The study presented an F-Measure to classify various events against the background noise. For the lowest-performing traffic and hammering events in the proposed approach, their system showed 0.87 for traffic with MFCC +3 feature set and 0.99 for horse-walking compared to 0.925 and 0.825 respectively in the proposed case for traffic and human walking. The higher accuracy of the proposed case may be attributed to the distinctly higher energy of horse-shoe sound compared to human steps on normal floor.

Similar work by Namgook and Kim [22] developed voice detection and classification modules integrating a total of 202 minutes of audio containing wood, metal, door slam and speech sounds via Gaussian mixture models (GMM) and support vector machines (SVM). GMM performed well giving an accuracy of $98.72 \%$ for "door-slamming + speech" activity compared to $88.3 \%$ for the closest presented case of "Door-closing DC + Traffic TF" in the proposed methodology. The lower accuracy in this case was possibly due to the "trailing/delayed states" of the DC event that were marred by the squeaking effect from the door hinges. Moreover, the entire door-closing duration often overlapped with the walking sound which may also have lead to poor WK detection accuracies.

Cristani [23] evaluated 66 audio-visual events and reported three audio cases predominantly covering the cases of room entries/exits, making calls, conversations, walking steps and button-presses. The overall discrimination accuracy between individuals entering an occupied and unoccupied lab was $62.12 \%$ compared to the proposed technique's accuracy of $74.55 \%$ for the noisy data under NARX/VRF model presented in Table 6.

Ntalampiras et al., [24] presented two sets of normal/abnormal audio events for general purpose security, smart home and ATM scenarios. The smart-home case contained 50.77 minutes from 492 of acoustic events covering events including normal speech, door bell, background noise, fire alarm and dropping of objects. The activities were evaluated against three probabilistic methods of GMM, SVM and HMM. The overall true detection rate for these three techniques and the three cases is further elaborated in Table 8. 
Table 6: NARX-based sub-event detection failure and CRF/HMM-based complete event recognition failure rates for noisy and stable sound data

\begin{tabular}{|c|c|c|c|c|c|c|c|c|c|c|c|c|}
\hline & & & & & & Sub & even & Reco & nnitio & Failu & & \\
\hline & & Activity & TER & SEC & TSE & SEF & $\mathrm{CS}$ & DO & WK & $\mathrm{DC}$ & US & $\mathrm{CEF}$ \\
\hline & & $\begin{array}{l}\text { CS->DO->WK- } \\
>\text { DC }\end{array}$ & 15 & 4 & 60 & 8 & 1 & 3 & 4 & 0 & & 5 \\
\hline & & $\begin{array}{l}\text { CS->DO->DC- } \\
>\text { WK }\end{array}$ & 10 & 4 & 40 & 7 & 0 & 2 & 5 & 0 & & 5 \\
\hline & $\underset{\widetilde{J}}{\tilde{E}}$ & CS->DO->DC & 5 & 3 & 15 & 2 & 0 & 0 & 0 & 2 & & 0 \\
\hline & อొ & US->US & 20 & 2 & 40 & 1 & & 0 & 0 & 0 & 1 & 1 \\
\hline & $\frac{\sqrt{2}}{2}$ & DO->WK->DC & 15 & 3 & 45 & 5 & & 2 & 3 & 0 & & 4 \\
\hline & Z & DO->DC->WK & 15 & 3 & 45 & 6 & & 2 & 4 & 0 & & 4 \\
\hline : & & $\begin{array}{l}\text { US->DO->WK- } \\
>\text { DC }\end{array}$ & 15 & 4 & 60 & 10 & & 1 & 3 & 2 & 4 & 6 \\
\hline $\begin{array}{l}-\frac{5}{5} \\
\frac{5}{0}\end{array}$ & & $\begin{array}{l}\text { US->DO->DC- } \\
>\text { WK }\end{array}$ & 15 & 4 & 60 & 7 & & 2 & 3 & 0 & 2 & 3 \\
\hline ț & & $\begin{array}{l}\text { CS->DO->WK- } \\
>\text { DC }\end{array}$ & 15 & 4 & 60 & 5 & 0 & 2 & 3 & 0 & & 3 \\
\hline 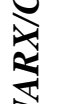 & & $\begin{array}{l}\text { CS->DO->DC- } \\
>\text { WK }\end{array}$ & 10 & 4 & 40 & 3 & 0 & 0 & 3 & 0 & & 3 \\
\hline & $\underset{\widetilde{J}}{\tilde{J}}$ & $\mathrm{CS}->\mathrm{DO}->\mathrm{DC}$ & 5 & 3 & 15 & 1 & 0 & 0 & 0 & 1 & & 0 \\
\hline & 0 & US->US & 20 & 2 & 40 & 0 & & 0 & 0 & 0 & 0 & 0 \\
\hline & $\frac{0}{\pi}$ & DO->WK->DC & 15 & 3 & 45 & 4 & & 2 & 2 & 0 & & 3 \\
\hline & & DO->DC->WK & 15 & 3 & 45 & 6 & & 3 & 3 & 0 & & 3 \\
\hline & & $\begin{array}{l}\text { US->DO->WK- } \\
>\text { DC }\end{array}$ & 15 & 4 & 60 & 7 & & 2 & 1 & 0 & 4 & 5 \\
\hline & & $\begin{array}{l}\text { US->DO->DC- } \\
>\text { WK }\end{array}$ & 15 & 4 & 60 & 9 & & 2 & 4 & 1 & 2 & 4 \\
\hline & & & & & & & & & & & & \\
\hline & & $\begin{array}{l}\text { CS->DO->WK- } \\
>\text { DC }\end{array}$ & 15 & 4 & 60 & 11 & 2 & 3 & 5 & 1 & & 6 \\
\hline & & $\begin{array}{l}\text { CS->DO->DC- } \\
>\text { WK }\end{array}$ & 10 & 4 & 40 & 8 & 1 & 1 & 6 & 0 & & 7 \\
\hline & 芯 & CS->DO->DC & 5 & 3 & 15 & 2 & 0 & 0 & 0 & 2 & & 2 \\
\hline & ค็ & US->US & 20 & 2 & 40 & 1 & & 0 & 0 & 0 & 1 & 1 \\
\hline$\cdot 8$ & $\frac{\widehat{a}}{0}$ & DO->WK->DC & 15 & 3 & 45 & 7 & & 2 & 3 & 2 & & 3 \\
\hline. & & DO->DC->WK & 15 & 3 & 45 & 8 & & 2 & 5 & 1 & & 5 \\
\hline$\frac{5}{0}$ & & $\begin{array}{l}\text { US->DO->WK- } \\
>\text { DC }\end{array}$ & 15 & 4 & 60 & 8 & & 1 & 2 & 2 & 3 & 5 \\
\hline$\underset{\vdots}{\vdots}$ & & $\begin{array}{l}\text { US->DO->DC- } \\
>\text { WK }\end{array}$ & 15 & 4 & 60 & 11 & & 2 & 3 & 4 & 2 & 4 \\
\hline 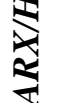 & & $\begin{array}{l}\mathrm{CS}->\mathrm{DO}->\mathrm{WK}- \\
>\mathrm{DC}\end{array}$ & 15 & 4 & 60 & 6 & 1 & 1 & 3 & 1 & & 3 \\
\hline & & $\begin{array}{l}\text { CS->DO->DC- } \\
>\text { WK }\end{array}$ & 10 & 4 & 40 & 6 & 0 & 0 & 4 & 2 & & 4 \\
\hline & $\frac{0}{2}$ & CS->DO->DC & 5 & 3 & 15 & 3 & 0 & 0 & 0 & 3 & & 3 \\
\hline & ज苟 & US->US & 20 & 2 & 40 & 0 & & & & 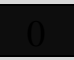 & 0 & 0 \\
\hline & & DO->WK->DC & 15 & 3 & 45 & 4 & & 2 & 1 & 1 & & 2 \\
\hline & & DO->DC->WK & 15 & 3 & 45 & 5 & & 1 & 2 & 2 & & 2 \\
\hline
\end{tabular}




\begin{tabular}{|l|l|l|l|l|l|l|l|l|l|l|l|}
\hline & $\begin{array}{l}\text { US->DO->WK- } \\
>\text { DC }\end{array}$ & 15 & 4 & 60 & 6 & 0 & 1 & 2 & 2 & 1 & 4 \\
\hline $\begin{array}{l}\text { US->DO->DC- } \\
>\text { WK }\end{array}$ & 15 & 4 & 60 & 10 & 0 & 1 & 5 & 3 & 1 & 5 \\
\hline
\end{tabular}

Table 7: Overall audio event recognition accuracy for CRF and HMM-based classifiers

\begin{tabular}{|l|r|}
\hline Total Events & \\
\hline Recorded Events & 220 \\
\hline Sub-events & 730 \\
\hline CRF Event Recognition Outcome & \\
\hline $\begin{array}{l}\text { Sub-Event Recognition Failure Rate } \\
\text { (ND) }\end{array}$ & $12.60 \%$ \\
\hline Complete Event Failure Rate (ND) & $25.45 \%$ \\
\hline $\begin{array}{l}\text { Sub-Event Recognition Failure Rate } \\
\text { (ND) }\end{array}$ & $9.59 \%$ \\
\hline Complete Event Failure Rate (ND) & $19.09 \%$ \\
\hline & \\
\hline HMM Event Recognition Outcome & $15.34 \%$ \\
\hline $\begin{array}{l}\text { Sub-Event Recognition Failure Rate } \\
\text { (ND) }\end{array}$ & $30.00 \%$ \\
\hline Complete Event Failure Rate (ND) & $10.96 \%$ \\
\hline $\begin{array}{l}\text { Sub-Event Recognition Failure Rate } \\
\text { (ND) }\end{array}$ & $20.91 \%$ \\
\hline Complete Event Failure Rate (ND) \\
\hline
\end{tabular}

Table 8: Comparison of work by Ntalampiras et al., [24] with the proposed technique comparing stable CEF values taken from Table 6

\begin{tabular}{|l|c|c|c|}
\hline & $\begin{array}{l}\text { GMM } \\
\text { Clustering }\end{array}$ & HMM & CRF \\
\hline Smart Home & 96.1 & - & - \\
\hline General Purpose Security & 96.4 & - & - \\
\hline ATM & 91.6 & - & - \\
\hline Card-based Entry (Table 6) & - & 79.09 & 80.91 \\
\hline
\end{tabular}

From Table 8, the difference of accuracies can generally be attributed to the difference-of-context in which the algorithms were applied and the datasets were recorded. Further work is necessary to evaluate the application of NARX/HMM and NARX/CRF models on specialist events described in the work of Ntalampiras et al., [24]. 


\section{Conclusion and Future Directions}

The principal aim of development of this architecture was to evaluate its ability to offer identification accuracies that could be integrated to an outdoor video and laser-range-finding solution for perimeter monitoring (Figure 1 and Figure 2). The work therefore presented the development of a test-bed platform to evaluate the ultimate development of an industrial-scale, two-phase discrete audio event detection and identification and time-series-based audio event recognition algorithm to be used in indoor/outdoor perimeter security settings. Therefore, the aim of the underlying research achieved an outlook into the susceptibility of autoregressive neural networks to process noisy sound data in the presence of support variables. As the system incorporated directional sound input, a marked improvement was noted in the identification accuracy when the NARX classifier was used. The overall accuracy further improved with a CRF model for time-series-based audio event classification.

A further analysis of the overall system architecture shown in Figure 3 fits into the capability of an industrial laser range-finding unit to pan in the direction indicated by the exogenous variable to create a 4D depth-map via a laser range finder. The 3D image thus obtained can then be utilized via a set of video-based computer vision algorithms to identify the type of activity in the particular area. Development of such a methodology will significantly eliminate blind-spot vulnerabilities in addition to minimizing the overall FAR. It must be noted that in the current state-of-the-art, the MS Kinect device used to develop indoor depth-map streams cannot be used under direct sunlight due to its infrared characteristics. This shortcoming presents a substantial amount of future work in the domain of outdoor 3D object cloud generation for gesture/behavioral profiling purposes.

\section{Acknowledgments}

The authors would like to offer their thanks to the Knowledge Transfer Partnerships/Innovate UK for their support. The project is a joint venture between STS Defence Ltd and University of Portsmouth. The baseline sound database utilized in this database is from the sounds courtesy of the Sound Events Database http://www.auditorylab.org/) Copyright 2008, Laurie M. Heller. Funding provided by NSF award 0446955.

\section{References}

[1] J. Pavón, J. Gómez-Sanz, A. Fernández-Caballero, and J. J. Valencia-Jiménez, "Development of intelligent multisensor surveillance systems with agents," Robotics and Autonomous Systems, vol. 55, pp. 892-903, 12/31/ 2007.

[2] F. Šuligoj, B. Šekoranja, M. Švaco, and B. Jerbić, "Object Tracking with a Multiagent Robot System and a Stereo Vision Camera," Procedia Engineering, vol. 69, pp. 968-973, // 2014.

[3] D. Lee, G. Kim, D. Kim, H. Myung, and H.-T. Choi, "Vision-based object detection and tracking for autonomous navigation of underwater robots," Ocean Engineering, vol. 48, pp. 59-68, 7// 2012.

[4] U. Antao, J. Choma, A. Dibazar, and T. Berger, "Low power, long life design for smart intelligence, surveillance, and reconnaissance (ISR) sensors," in Homeland Security (HST), 2012 IEEE Conference on Technologies for, 2012, pp. 631-636.

[5] G. Janssens-Maenhout, F. De Roo, and W. Janssens, "Contributing to shipping container security: can passive sensors bring a solution?," Journal of Environmental Radioactivity, vol. 101, pp. 95-105, 2// 2010.

[6] H. Wu, Y. Rao, C. Tang, Y. Wu, and Y. Gong, "A novel FBG-based security fence enabling to detect extremely weak intrusion signals from nonequivalent sensor nodes," Sensors and Actuators A: Physical, vol. 167, pp. 548-555, 6// 2011. 
[7] B. Dong, J. Hao, and V. Paulose, "Armored-cable-based FBG security fence for perimeter intrusion detection with higher performance," Sensors and Actuators A: Physical, vol. 180, pp. 15-18, 6// 2012.

[8] S. Yusuf, Adnan, D. Brown, J, A. Mackinnon, and R. Papanicolaou, "A Pixel-level, Intensitybased Nonlinear Autoregressive Classifier (NARX) with Chromatic Exogenous Input for Efficient Image Background Subtraction," presented at the International Joint Conference on Neural Networks, Dallas, Texas, 2013.

[9] S. Yusuf, Adnan, S. Shetty, N. Wilkinson, and D. Brown, "A Modified Codebook-based Background Subtraction Technique to improve Activity Classification in Highly Variable Environments," in International Conference on Industrial Engineering, Dubai, UAE, 2012.

[10] K. Kim, T. H. Chalidabhongse, D. Harwood, and L. Davis, "Real-time foregroundbackground segmentation using codebook model," Real-Time Imaging, vol. 11, Jun 2005.

[11] N. Buch, S. A. Velastin, and J. Orwell, "A Review of Computer Vision Techniques for the Analysis of Urban Traffic," Intelligent Transportation Systems, IEEE Transactions on, vol. 12, pp. 920-939, 2011.

[12] J. Candamo, M. Shreve, D. B. Goldgof, D. B. Sapper, and R. Kasturi, "Understanding Transit Scenes: A Survey on Human Behavior-Recognition Algorithms," Intelligent Transportation Systems, IEEE Transactions on, vol. 11, pp. 206-224, 2010.

[13] R. Chang, C. Teck Wee, K. Leman, W. Hee Lin, and Z. Jie, "Automatic cooperative camera system for real-time bag detection in visual surveillance," in Distributed Smart Cameras (ICDSC), 2013 Seventh International Conference on, 2013, pp. 1-6.

[14] X. Kang, G. Ogunmakin, L. Yue, P. A. Vela, and W. Yongtian, "PTZ camera-based adaptive panoramic and multi-layered background model," in Image Processing (ICIP), 2011 18th IEEE International Conference on, 2011, pp. 2949-2952.

[15] J. L. A. Herrera and C. Xiang, "Online configuration of PTZ camera networks," in Distributed Smart Cameras (ICDSC), 2012 Sixth International Conference on, 2012, pp. 1-6.

[16] H. Jwu-Sheng, S. Tzung-Min, H. Heng-Chia, and L. Pei-Ching, "Vision-based Indoor Scene Cognition Using a Spatial Probabilistic Modeling Method," in Automation Science and Engineering, 2006. CASE '06. IEEE International Conference on, 2006, pp. 620-625.

[17] L. Tsungnan, B. G. Horne, P. Tino, and C. L. Giles, "Learning long-term dependencies in NARX recurrent neural networks," Neural Networks, IEEE Transactions on, vol. 7, pp. 13291338, 1996.

[18] J. Portelo, M. Bugalho, I. Trancoso, J. Neto, A. Abad, and A. Serralheiro, "Non-speech audio event detection," in Acoustics, Speech and Signal Processing, 2009. ICASSP 2009. IEEE International Conference on, 2009, pp. 1973-1976.

[19] B. S. Atal and J. Remde, "A new model of LPC excitation for producing natural-sounding speech at low bit rates," in Acoustics, Speech, and Signal Processing, IEEE International Conference on ICASSP '82., 1982, pp. 614-617.

[20] M. Sarfraz, Y. A. Syed, and M. Zeeshan, "A system for sign language recognition using fuzzy object similarity tracking," in Information Visualisation, 2005. Proceedings. Ninth International Conference on, 2005, pp. 233-238.

[21] M. K. Geetha and S. Palanivel, "HMM Based Automatic Video Classification Using Static and Dynamic Features," in Conference on Computational Intelligence and Multimedia Applications, 2007. International Conference on, 2007, pp. 277-281.

[22] C. Namgook and K. Eun-Kyoung, "Enhanced voice activity detection using acoustic event detection and classification," Consumer Electronics, IEEE Transactions on, vol. 57, pp. 196202, 2011.

[23] M. Cristani, M. Bicego, and V. Murino, "Audio-Visual Event Recognition in Surveillance Video Sequences," Multimedia, IEEE Transactions on, vol. 9, pp. 257-267, 2007.

[24] S. Ntalampiras, I. Potamitis, and N. Fakotakis, "Probabilistic Novelty Detection for Acoustic Surveillance Under Real-World Conditions," Multimedia, IEEE Transactions on, vol. 13, pp. 713-719, 2011. 
Dr Syed Yusuf did his MS in Computer Science from King Fahd University of Petroleum \& Minerals, Saudi Arabia. His major research focus has been on machine learning algorithms for gesture recognition and intelligent transport systems. He completed his $\mathrm{PhD}$ from the School of Technology, University of Wolverhampton and then his Post Doc from the London Metropolitan University's Faculty of Life Sciences and Computing.

Dr Yusuf currently works as a Research Scientist with STS Defence Ltd over a 2.3m project funded by the Innovate UK, with University of Portsmouth, Carnival, Satellite Applications Catapult, Merlin Ltd, University of Lancaster, National Oceanography Centre and the University of Southampton as partners. He is also a Microsoft Certified Professional and a C\# Technology Specialist (MCTS). He is currently working on next-generation inertial navigation and LiDAR-based scanning devices to develop dead-reckoning systems for firstresponders in GPS-denied search and rescue environments. 
Professor David Brown joined the University of Portsmouth in 1998, having previously worked in industry as the Managing Director of an automation and diagnostics company, Technical Director within Cambridge Electronic Industries and a lecturer at the University of Southampton. He is now the Director of the Centre of Intelligent Data Solutions, undertaking a number of concurrent research projects. Over the past two years the Institute team has attracted nearly $£ 2,000,000$ in funding from commercial research partners.

Prof Brown direct teams in the areas of data analysis with fault diagnostics and prognostics, autonomous vehicles and resource management, intelligent robotics, image processing and monitoring systems and ambient intelligence. 
Mr Alan Mackinnon has an MBA with the Portsmouth Business School, University of Portsmouth and a Bachelor of Science (Honours) with the Open University.

He is currently working as the Business Manager of the Engineering Services division at STS Defence Ltd. Mr Mackinnon has a 30-year-career encompassing his role as a POWEA Communication System Engineer with the Royal Navy, UHF Satcomm System Engineer and a Project Manager.

His research interests include industrial artificial intelligence (AI), short-haul RF and longhaul 3G/GPRS communication systems in the domain of industrial condition monitoring and fire $\&$ rescue services. 


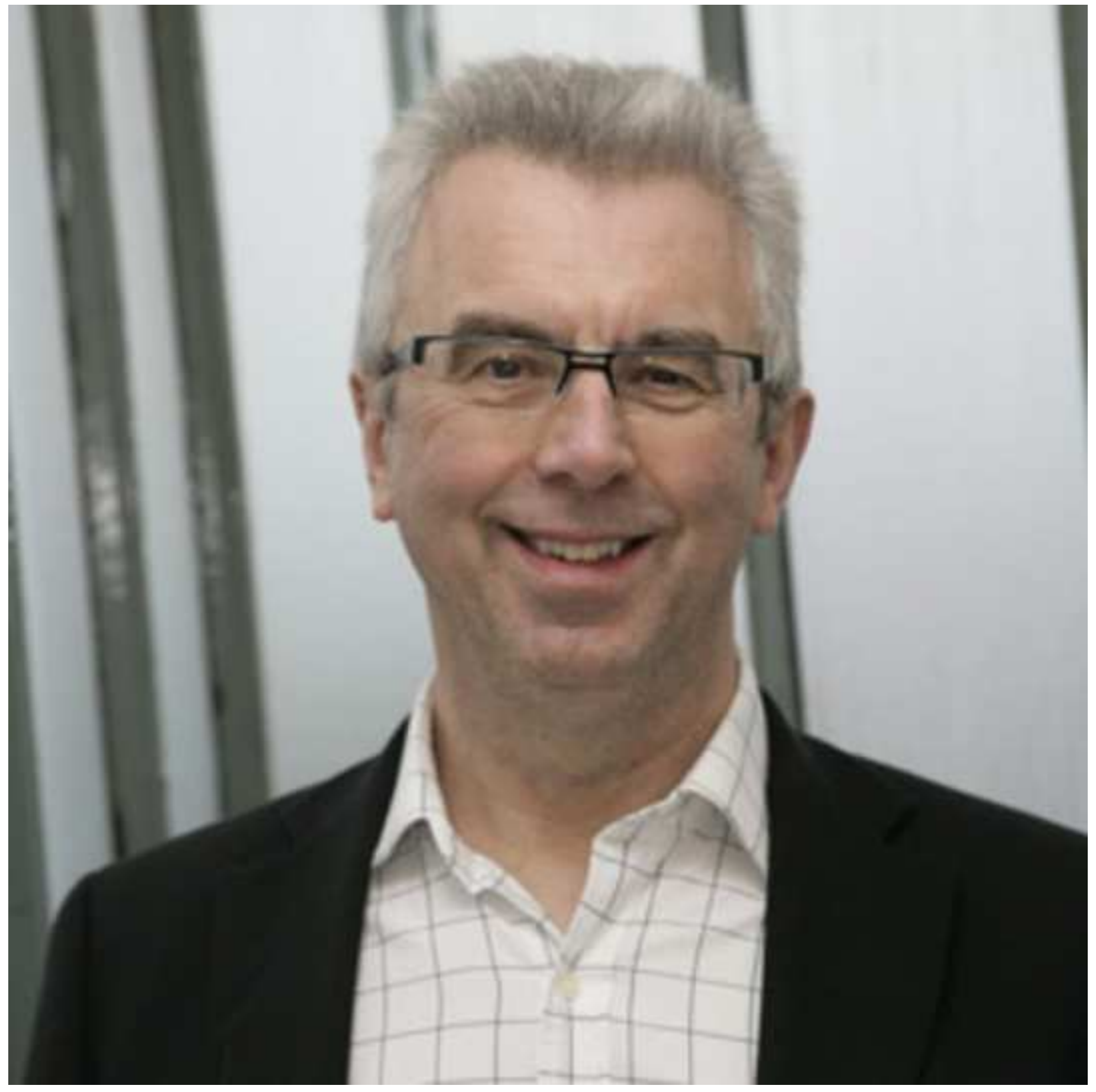




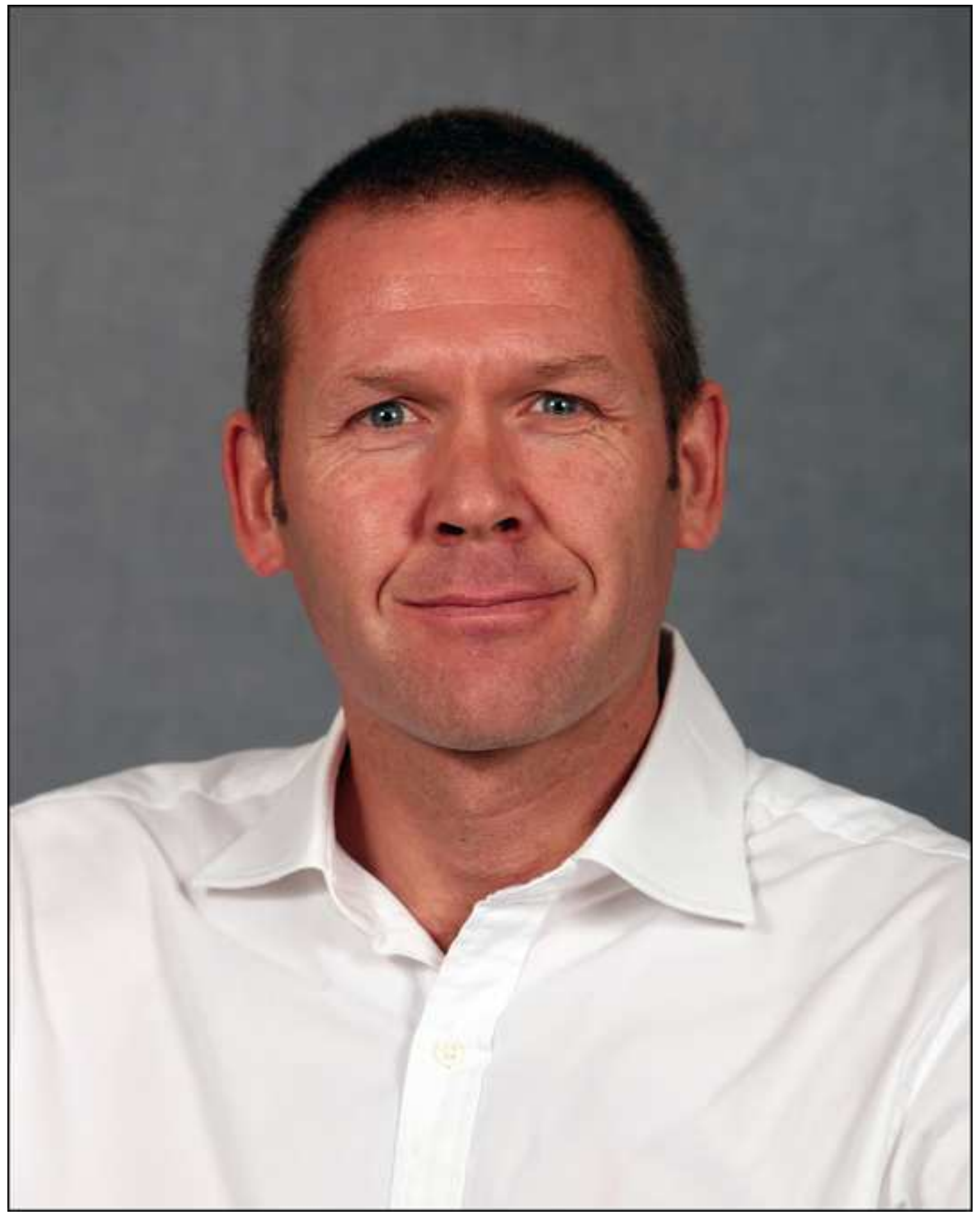

